\title{
Focusing Properties of High-Order Harmonics
}

\author{
Maria Hoflund $\mathbb{D},{ }^{1}$ Jasper Peschel, ${ }^{1}$ Marius Plach, ${ }^{1}$ Hugo Dacasa $\mathbb{D}^{1},{ }^{1}$ Kévin Veyrinas $\mathbb{D},{ }^{2}$ \\ Eric Constant $\mathbb{D},{ }^{3}$ Peter Smorenburg, ${ }^{4}$ Hampus Wikmark $\mathbb{D}^{1},{ }^{1}$ Sylvain Maclot $\mathbb{D}^{1}{ }^{1}$

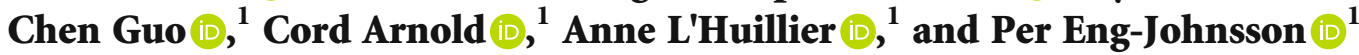 \\ ${ }^{1}$ Department of Physics, Lund University, P.O. Box 118, 22100 Lund, Sweden \\ ${ }^{2}$ Université de Bordeaux, CNRS, CEA, Centre Laser Intenses et Applications (CELIA), 43 Rue P. Noailles, 33400 Talence, France \\ ${ }^{3}$ Univ Lyon, Univ Claude Bernard Lyon 1, CNRS, Institut Lumière Matière, F-69622 Villeurbanne, France \\ ${ }^{4}$ ASML Research, ASML Netherlands B.V., 5504 DR Veldhoven, Netherlands
}

Correspondence should be addressed to Maria Hoflund; maria.hoflund@fysik.lth.se

Received 29 April 2021; Accepted 27 July 2021; Published 29 August 2021

Copyright (c) 2021 Maria Hoflund et al. Exclusive Licensee Xi'an Institute of Optics and Precision Mechanics. Distributed under a Creative Commons Attribution License (CC BY 4.0).

\begin{abstract}
Many applications of the extreme ultraviolet (XUV) radiation obtained by high-order harmonic generation (HHG) in gases require a small focus area in order to enable attosecond pulses to reach a high intensity. Here, high-order harmonics generated in Ar with a multiterawatt laser system in a loose focusing geometry are focused to a few micrometers using two toroidal mirrors in a Wolter configuration with a high demagnification factor. Using a knife-edge measurement technique, we determine the position and size of the XUV foci as a function of harmonic order. We show that the focus properties vary with harmonic order and the generation conditions. Simulations, based on a classical description of the harmonic dipole phase and assuming that the individual harmonics can be described as Gaussian beams, reproduce the experimental behavior. We discuss how the generation geometry affects the intensity and duration of the focused attosecond pulses.
\end{abstract}

\section{Introduction}

High-order harmonic generation (HHG) is a highly nonlinear process that converts intense low-frequency light into attosecond pulse trains in the extreme ultraviolet (XUV) spectral region. In spite of a low conversion efficiency [1-3], the choice of appropriate gas targets combined with the use of loose focusing geometries has led to high pulse energies on the order of microjoules [4-8]. The high intensity that can potentially be achieved by focusing this radiation opens the way to multiphoton ionization processes in the XUV range [7,9-12], and in particular to nonlinear XUV pump-XUV probe studies with attosecond resolution [13-15]. While XUV and X-ray radiation from free-electron lasers provide the required intensities for these types of studies [16, 17], they have only recently started to demonstrate the generation of attosecond pulses [18]. Using these sources for experiments is still very challenging, whereas HHG-based sources already provide a mature technology for nonlinear pump-probe experiments in the attosecond range.
To obtain both high intensity and attosecond pulse duration in the interaction with a low-density gas target, it is important to focus the broadband radiation on a small spot. This requires focusing the different harmonic components at the same position. Focusing of high-order harmonics has been achieved using ellipsoidal mirrors $[19,20]$, pairs of toroidal mirrors at grazing incidence [21-23], or spherical mirrors at normal incidence for low photon energy $[8,24]$. Focal spot sizes of the order of a few $\mu \mathrm{m}$ for the emitted harmonics in a certain spectral range are typically obtained. The methods used for measuring these spot sizes vary from direct techniques like the knife-edge method [20, 25-27] or microscopy [21] to indirect ones based on wavefront determination [23, 28-30]. Recently, focus sizes of the order of $0.3 \times 0.4 \mu \mathrm{m}^{2}$ have been measured for harmonics between 10 and $20 \mathrm{~nm}$ (i.e., $60-120 \mathrm{eV}$ ) focused with an ellipsoidal mirror [31].

Previous studies [30, 32-36] have shown that high-order harmonics have different wavefronts at the exit of the nonlinear medium, which can be convergent or divergent [35], corresponding to a real or virtual focus, respectively. The 
wavefront curvature of the harmonics is the sum of that of the driving laser and an additional contribution originating from the process behind the generation of high-order harmonics. The resulting harmonic radius of curvature and spatial profile [37] depends on harmonic order, laser intensity, and generation position. When they are refocused, this results in separated waist positions along the propagation direction, thus affecting the focusing properties of the harmonics and the peak intensity and pulse duration of the focused attosecond pulses. Although theoretically predicted in $[34,35]$, and experimentally shown for the total harmonic beam in [38], no experiments have yet quantitatively examined the influence of the generation geometry on the focus position, waist, and intensity of individual harmonics and attosecond pulses.

In the present study, we generate high-order harmonics in argon using a $45 \mathrm{~mJ}, 40 \mathrm{fs}$, Ti:Sapphire laser in a loose focusing geometry. We refocus the XUV radiation with a pair of toroidal mirrors in a Wolter configuration $[23,39]$ in order to achieve broadband focusing with a high demagnification factor. A knife-edge technique [20, 25-27] is used to measure the individual harmonic focus positions for different locations of the gas target relative to the laser focus in the generation chamber. In some cases, the harmonics are found to be refocused at positions that evolve strongly with the harmonic order. The observed experimental trends can be explained by a simple model which takes into account the intensity dependence of the spectral phase of the attosecond pulses, also called the dipole phase $[34,35]$. We find that the chromatic aberrations induced by the dipole phase affect strongly the temporal profile and intensity of the focused attosecond pulses.

\section{Experimental Method}

The experimental setup used in this study is sketched in Figure 1. We use a multiterawatt Ti:Sapphire laser system delivering $40 \mathrm{fs}, 806 \mathrm{~nm}$, and $45 \mathrm{~mJ}$ pulses [7]. A deformable mirror (DM), used together with an infrared wavefront sensor, allows us to correct for spatial aberrations and to control the IR focusing position with respect to the generation medium by introducing a slight curvature to the beam. After aberration correction, the measured laser wavefront has an RMS value of about $\lambda / 40$, corresponding to a Strehl ratio of 0.97. After going through an iris, the infrared beam is loosely focused with an $8.7 \mathrm{~m}$ spherical mirror into a $1 \mathrm{~cm}$ long, pulsed, Ar gas cell, generating high-order harmonics up to $45 \mathrm{eV}$. We estimate that the IR waists are approximately $340-360 \mu \mathrm{m}$ with corresponding Rayleigh lengths of 45 to $50 \mathrm{~cm}$ for the different focal lengths used in the experiment.

The XUV radiation is reflected, at a $10^{\circ}$ grazing incidence angle, by a fused silica (FS) plate, which is antireflectioncoated for the IR, and spectrally filtered by a $200 \mathrm{~nm}$ Al filter. The FS plate and Al filter allow us to completely eliminate the IR. Further, the broadband beam is refocused by Wolter optics, consisting of a prealigned assembly of two toroidal mirrors from Thales SESO, designed to image the generation medium, after $6 \mathrm{~m}$ propagation, with a high demagnification factor and minimal aberrations in the XUV range. The graz-

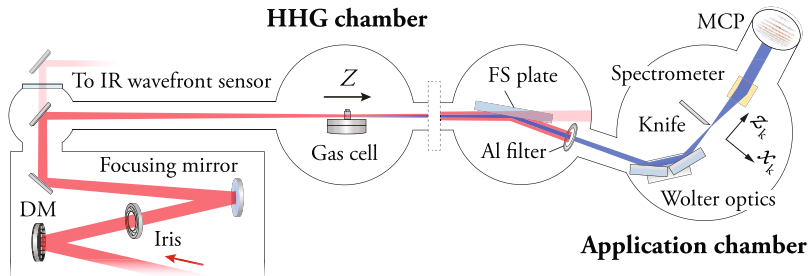

Figure 1: Schematic representation of the experimental setup. The spatially filtered IR beam (red) is first wavefront-corrected by a deformable mirror before it goes through an iris and is reflected by a focusing mirror. High-order harmonics (blue) are generated in an argon gas cell. The IR is filtered out by an IR antireflectioncoated fused silica plate followed by an aluminum filter. In the application chamber, the harmonic beam is focused by the Wolter optics. The knife is placed around the focus, and the beam is detected by a flat-field spectrometer.

ing incidence angle on the two toroidal mirrors is $15^{\circ}$, giving a total deviation angle of $60^{\circ}$. The Wolter optics assembly is aligned in our beamline by using a homemade XUV Hartmann wavefront sensor. The tangential focal lengths are $265.3 \mathrm{~mm}$ and $545.2 \mathrm{~mm}$ for the first and second mirror while the sagittal focal lengths are $265.0 \mathrm{~mm}$ and $544.4 \mathrm{~mm}$. The combined focal length of $164.2 \mathrm{~mm}$ provides a demagnification factor of 35. A homemade flat-field spectrometer, based on a Hitachi aberration-corrected concave grating, images the focus in the vertical direction onto a microchannel plate (MCP). In this configuration, no entrance slit is needed and the entire beam is characterized. The grating disperses the harmonics along the vertical axis of the MCP, while the far field spatial profile is recorded along the horizontal axis.

For each position of the cell relative to the IR focus along the propagation direction $Z$, called "generation position," we measure the focus position of the harmonics in the application chamber. Harmonic spectra are recorded for different $x_{\mathrm{k}}$ (transverse to the beam) and $z_{\mathrm{k}}$ (along the beam) positions of a knife-edge located close to the focus (see Figure 1). A rough estimate of the focus position for each harmonic can be obtained by looking at the diffraction image position of the knife-edge on the spatial profile recorded by the spectrometer. If the knife is after the focus, the shadow is on the same side as the knife, while if the focus is after, it is on the opposite side. This is illustrated in Figure 2, showing spectra obtained without the knife-edge (a) and for three positions of the knife-edge (b-d). In (b) (respectively, d), the knife is before (after) the focus for all harmonic orders and the shadow is observed on the right (left) sides of the profile. In (c), the shadow of the knife is on the right side for the lower-order harmonics while it is on the left side for the higher-order harmonics. This means that the knife is before the focus for the lower-order harmonics, and after the focus for the higher orders. The harmonics in the middle are less affected by the knife, which indicates that it is located close to their focus. These results show that the harmonics are indeed focused at different positions along the propagation axis. 


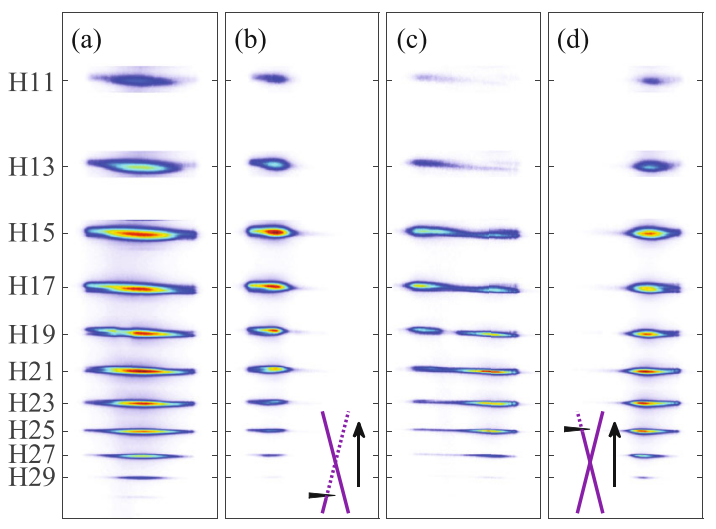

FIGURE 2: Measured spectra with no knife (a), the knife before (b) and after (d) the focus, and the knife in the focal region (c). The second-order diffraction from the spectrometer grating is numerically filtered out. The insets in (b) and (d) illustrate the position of the knife relative to the focus and its shadow. The knife positions in (b-d) are $z_{\mathrm{k}}=-8.3, z_{\mathrm{k}}=0 \mathrm{~mm}$, and $z_{\mathrm{k}}=9.1 \mathrm{~mm}$.

Mapping out the individual harmonic focus positions requires scanning both $x_{\mathrm{k}}$ and $z_{\mathrm{k}}$ in small steps. Instead, we use a faster and less tedious method based on measurements at only four positions in $z_{\mathrm{k}}$, outside the Rayleigh range $\left(z_{\mathrm{R}} \approx 1 \mathrm{~mm}\right)$, two before and two after the focus, which is sufficient to extract the position of each focus. Figure 3 illustrates this method for the $21^{\text {st }}$ harmonic. The harmonic intensity as a function of knife insertion $\left(x_{\mathrm{k}}\right)$ is recorded for each knife-edge position along the propagation direction $\left(z_{\mathrm{k}}\right)$ (see Figure 3(a)). Assuming Gaussian beams, this data is then fitted to a normalized error function, $f\left(x_{\mathrm{k}}\right)=\left\{\operatorname{erf}\left[b\left(x_{\mathrm{k}}-c\right)\right]+1\right\} / 2$, representing the integral of a Gaussian function $\exp \left[-b^{2}(x-c)^{2}\right]$ from $-\infty$ to $x_{\mathrm{k}}$. For each knife-edge position, the $1 / e^{2}$ beam waist radius $\left(w_{q}=\sqrt{2} / b\right)$ is obtained from the fit. The position of the harmonic focus $\left(z_{q}\right)$ can be determined by performing a linear fit of the beam widths, after mirroring those corresponding to the knife-edge position behind the focus (Figure 3(b)). If we assume that the harmonics are ideal Gaussian beams with $\mathbb{M}^{2}=1$, the beam waists $\left(w_{q}\right)$ can also be estimated as $\lambda_{q} /\left(\pi \theta_{q}\right)$, where $\lambda_{q}$ is the wavelength of the $q^{\text {th }}$ harmonic and $\theta_{q}$ the (half) divergence angle. Note that $w_{q}$ represents a lower limit to the beam waist and that a better estimation would require a measurement of the quality factor $\mathbb{M}^{2}$, which has not been done in the present work.

\section{Theoretical Model}

HHG can be described by a three-step process: tunneling ionization, electron acceleration by the laser field, and recombination $[40,41]$. In the second step, the electron wave packet accumulates a phase, called the dipole phase, which is then transferred to the harmonic field. Two families of trajectories, related to the time spent by the electron in the continuum ("short" or "long"), have been identified and observed experimentally $[42,43]$. The dipole phase is often expressed as $\alpha I$,

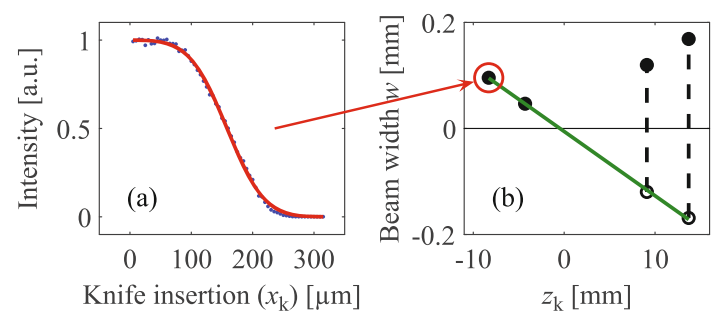

FIgURE 3: Illustration of our analysis method for one harmonic order (21). (a) Normalized harmonic intensity as a function of knife-edge insertion (blue dots). An error function is fitted to the data (red curve), allowing us to determine the beam width at $1 / e^{2}$, marked with a red circle in (b). (b) Measured beam widths for four different knife-edge positions along the propagation direction (black dots). A linear fit (green curve) is performed between these widths, after mirroring two of them relative to the horizontal axis (black circles). The focus is the position where the fit crosses zero.

where $\alpha$ depends on the harmonic order and electron trajectory, and $I$ is the intensity of the driving laser [44]. This expression is derived by using the strong-field approximation to solve the time-dependent Schrödinger equation (TDSE) of the single-atom response. Instead, we use an analytical expression derived by solving Newton's equation of motion $[34,45]$ :

$$
\Phi_{i}(q, I)=\alpha_{i} I+t_{p i}\left(q \omega-\omega_{p}\right)+\frac{\gamma_{i}}{I}\left(q \omega-\omega_{p}\right)^{2}
$$

where $i$ refers to the trajectory (short or long), $q$ is the harmonic order, $I$ and $\omega$ are the fundamental intensity and angular frequency, respectively, $\omega_{p}$ is the angular frequency corresponding to the ionization energy, and $t_{p i}$ is a return time, as explained in [34]. We here consider only the contribution from the short trajectory, which is mainly observed in our experiment. In Equation (1), the quantity $\alpha_{i}$ is independent of the harmonic order and is equal to zero for the short trajectory, while the quantity $\gamma_{i}$ is equal to $1.03 \times 10^{-18} \mathrm{~s}^{2}$ $\mathrm{W} \mathrm{cm}{ }^{-2}$ for a driving laser of $800 \mathrm{~nm}$. The first and third terms depend on the laser intensity, leading to an orderdependent wavefront curvature.

We assume that high-order harmonics are generated at position $Z$ relative to the IR focus (located at $Z=0$ ) in a thin medium [46]. For a Gaussian fundamental beam, with a profile $I_{0} \exp \left[-2 r^{2} / w^{2}(Z)\right]$, where $I_{0}$ is the peak intensity, $r$ is the radial coordinate, and $w(Z)$ is the beam width at position $Z$, the $r$-dependence of the dipole phase can be approximated by

$$
-\frac{2 \alpha_{i} I_{0} w_{0}^{2}}{w^{4}(Z)} r^{2}-\frac{2 \gamma_{i}\left(q \omega-\omega_{p}\right)^{2}}{I_{0} w_{0}^{2}} r^{2}
$$

The generated harmonic field also includes an $r$-dependent phase contribution from the fundamental, which is equal to $q k r^{2} / 2 R(Z)$, where $k$ is the fundamental wavevector and $R(Z)$ is its radius of curvature. The total radius of 

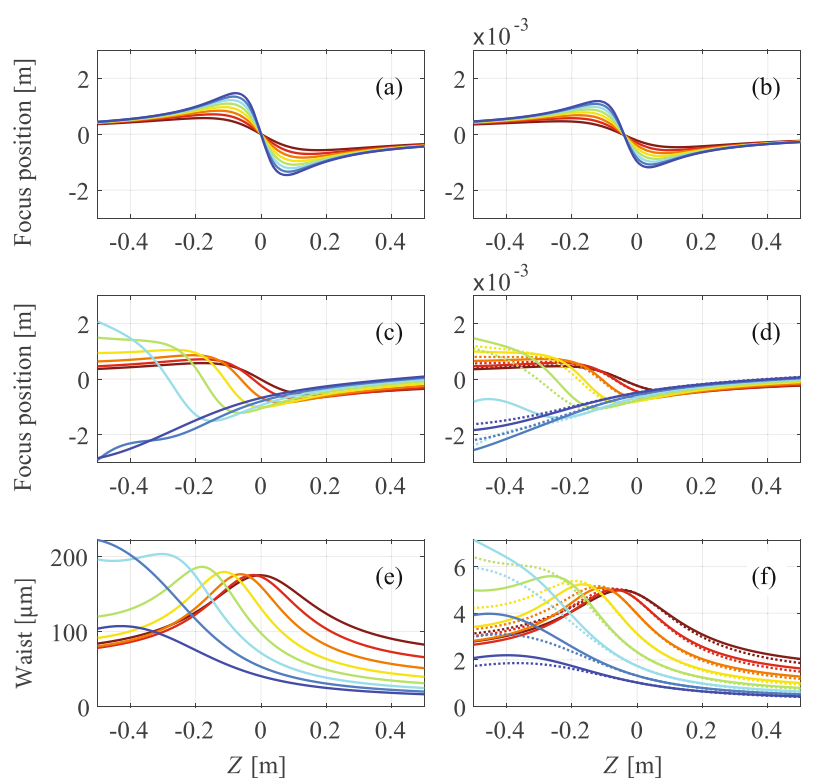

Figure 4: Calculated harmonic focus positions as a function of generation position $Z$, in the HHG chamber $(\mathrm{a}, \mathrm{c})$ and after being refocused, in the application chamber $(b, d)$. In $(e, f)$, the corresponding waists are shown. The simulation is run without (a, b) and with (c-f) the dipole phase included. The harmonic order goes from 11 (red) to 25 (blue). The dashed lines in (d) and (f) simulate the experimental conditions, where the relative mediumlaser focus distance is varied by changing the focal length of the generating laser with a deformable mirror.

curvature of the harmonic field, corresponding to one trajectory $i$, is given by

$$
\frac{1}{R_{i}(Z)}=\frac{1}{R(Z)}-\frac{4 \alpha_{i} I_{0} w_{0}^{2} c}{w^{4}(Z) q \omega}+\frac{4 \gamma_{i}\left(q \omega-\omega_{p}\right)^{2} c}{I_{0} w_{0}^{2} q \omega}
$$

where $c$ is the speed of light and $w_{0}$ is the fundamental beam waist [34].

To fully characterize the harmonic beams, the width at the generation position is estimated by assuming that the harmonic intensity $\left(I_{q}\right)$ is proportional to the fundamental intensity to the power of $p\left(I_{q} \propto I_{0}^{p}\right)$ [46-50], where the degree of nonlinearity, $p$, is assumed to be equal to 4 , which is confirmed by TDSE calculations [34]. The harmonic width, at the position of the gas target, is then simply half the width of the driving laser. Assuming Gaussian optics for the harmonic beam, knowing the width and radius of curvature at the generation position $Z$ allows us to determine the beam properties at any position.

Figure 4 shows the calculated focus positions and waist size, with respect to the IR focus, for harmonic 11 to 25 as a function of generation position $Z$. The left column shows the (virtual or real) focus positions and waists at generation while the right column shows the foci and waists of the refocused beams. The first row $(a, b)$ shows results obtained without the dipole phase. In this case, the harmonics have the same wavefront, which is equal to that of the fundamental. When they are generated at the IR waist, they have the same focus, which is identical to the IR focus (Figure 4(a)). However, for this generation position $(Z=0)$, the harmonics are not refocused at the same focus position in the application chamber (Figure 4(b)). In this very loose focusing geometry, some harmonics have very long Rayleigh lengths. Consequently, the focusing optics is not fully in the far field, so that the curvatures of the harmonics at this position are slightly harmonic-dependent. Therefore, the harmonics will be focused at slightly different positions. In this case, where the dipole phase is not included, the foci in the application chamber are overlapped for all harmonics, when the generation medium is slightly before the IR focal spot. This is a clear deviation from the predictions of geometrical optics, due to the long Rayleigh lengths mentioned above.

The second row $(c, d)$ is calculated including the dipole phase. This leads to an asymmetric focus position distribution for positive and negative generation positions. Furthermore, there is no generation position where all harmonics are focused at the same position. We also show in $(e, f)$ the harmonic waists both in the generation and application chambers, including the dipole phase in the calculations.

Finally, we also simulate the experimental conditions, where the cell position is fixed and where the focal length is slightly varied in order to change the relative position between the generation and the IR focus. As expected, the results, shown as dashed lines in Figures 4(d) and 4(f), present the same qualitative behavior as when the focal length is not varied (solid lines), and only minor quantitative differences. The simulation with the varying focal length is used in the comparison with the experimental results.

\section{Results}

To describe our experimental geometry, where the generation cell is fixed and the position of the IR focus varied, we introduce $\Delta Z=Z_{\mathrm{m}}-Z_{0}$, which is the medium position $\left(Z_{\mathrm{m}}\right)$ relative to the IR focus $\left(Z_{0}\right)$. Similarly, in the application chamber, we refer to the refocused focus positions in the coordinate $\Delta z=z_{q}-z_{0}$, where $z_{0}$ is the position of the refocused IR, which was estimated from the simulations. The $19^{\text {th }}$ harmonic is removed from the dataset because its position on the detector coincides with a damaged area on the microchannel plate of the spectrometer, making its detection unreliable.

The results of our knife-edge measurements are shown in Figures 5(a)-5(e), for different generation positions, as shown at the top of the figure. The experimental data are compared with simulations (dashed line) using the model presented above. The different harmonics are found to be focused at different positions, with a separation of up to $1.5 \mathrm{~mm}$, which is significant since the harmonic Rayleigh lengths are typically around $1 \mathrm{~mm}$. This effect decreases as the generation position moves towards and beyond the laser focus. When the medium is before the focus (see Figures 5(a)-5(c)), the lower-order harmonics are focused approximately one $\mathrm{mm}$ after the higher-order harmonics, on different sides of the IR focus. When the generation medium is centered at the infrared focus or beyond (see Figures 5(d) and 5(e)), all of the harmonics are focused much 
(a)

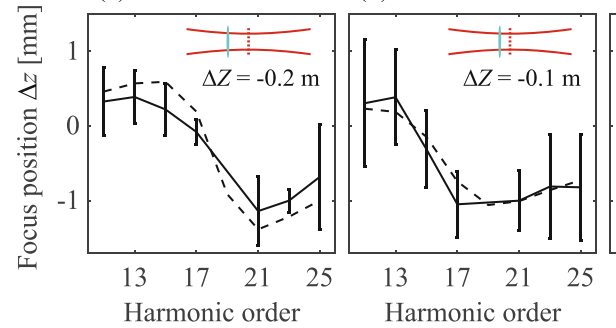

(c)

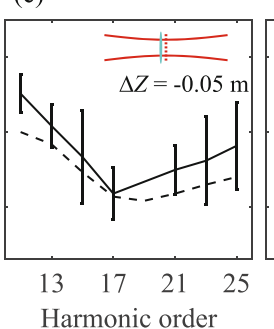

(d)

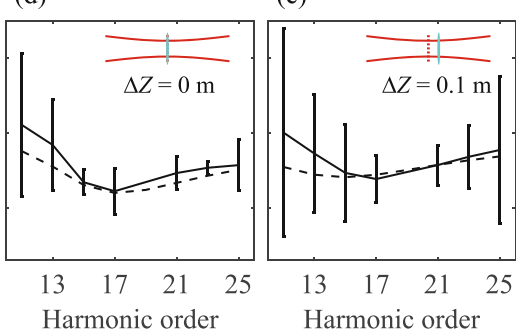

FIGURE 5: Measured (solid line) and simulated (dashed line) focus positions of harmonics 11-25 for five different generation positions in relation to the IR focus. The insets indicate the geometry of the generation in the five cases. The medium is represented by the vertical blue line, while the position of the IR focus is indicated in red. The error bars are obtained from the standard deviation of the fit performed in Figure 3.
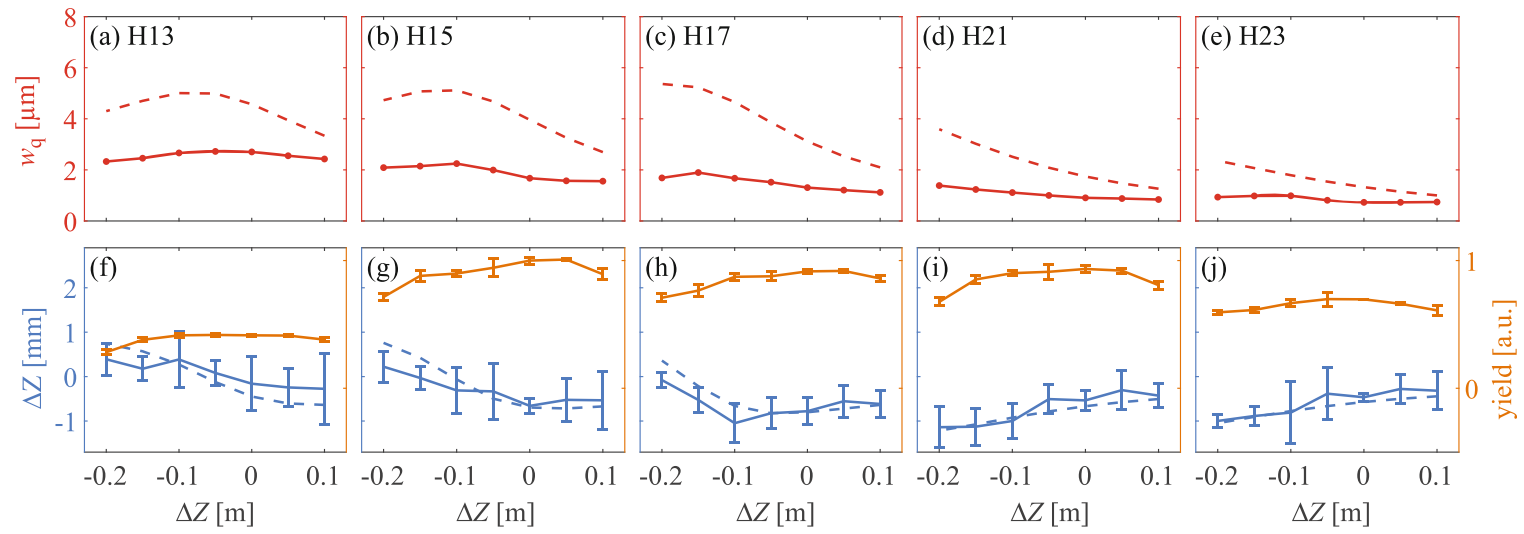

Figure 6: Simulated (dashed red) and measured waist size (solid red) as a function of generation position, for harmonic $13,15,17,21$, and 23 (a-e). Measured (solid blue) and simulated (dashed blue) focus positions, and measured yield (orange) as a function of generation position for the same harmonic orders $(\mathrm{f}-\mathrm{j})$.

closer to each other, at a position before the IR focus. Simulations reproduce the main features of the experimental data, which indicates that, despite its simplicity, our model describes the physics behind the focusing properties of high-order harmonics well [34, 35].

In Figures 6(a)-6(e), we present the measured (solid line) and simulated (dashed line) beam waists $\left(w_{q}\right)$, for a few harmonics $(13,15,17,21$, and 23), as a function of generation position. As explained in the experimental section, the measured beam waists should be considered lower limits. We find that the harmonic waist sizes decrease with order. For harmonics 13,15 , and $17(\mathrm{a}-\mathrm{c})$, the generation position leading to the maximum waist moves progressively from around $\Delta Z=0$ to negative values. The measured values for the harmonic beam waists are consistent with (but slightly below) previous spectrally integrated measurements, which were performed by wavefront analysis of the total beam combined with numerical back-propagation and gave $3.6 \times 4$ $\mu \mathrm{m}^{2}$ FWHM $\left(3.1 \times 3.4 \mu \mathrm{m}^{2}\right.$ half-width at $\left.1 / e^{2}\right)$ [23]. The simulations in Figure 6 (a-e, dashed lines) follow qualitatively the experimental results, with slightly larger waists. This difference might be due to a deviation of the experimental laser (and consequently harmonic) beams from a Gaussian behavior (i.e., $\mathbb{M}^{2}>1$ ). Another possible cause of the discrepancy between the experimental results and our simulations could be that the dipole phase is more complex than the model dipole or that phase-matching impacts also the wavefront of the XUV beam. Despite the approximation of our model, the agreement is very satisfactory.

Figures 6(f)-6(j) show the focus positions (blue) and yield (orange) for the same harmonics, as a function of generation position. Simulations, indicated by dashed lines, agree well with the experimental measurements. As shown in Figure 5, the harmonic focus position varies with the generation geometry, especially for the lower-order harmonics. The harmonic yield, on the other hand, is relatively insensitive to the generation position over the studied range.

\section{Discussion}

Our results can be used to estimate not only the intensity of the refocused individual harmonics but also that of the attosecond pulses in the train, in which case the relative phase of the harmonics has to be included [8]. The electric field describing the attosecond pulse train can be expressed as

$$
E(t, r, z)=\sum_{q} \sqrt{I_{q}(t, r, z)} \exp \left(i q \omega t+i \phi_{q}(t, r, z)\right)
$$



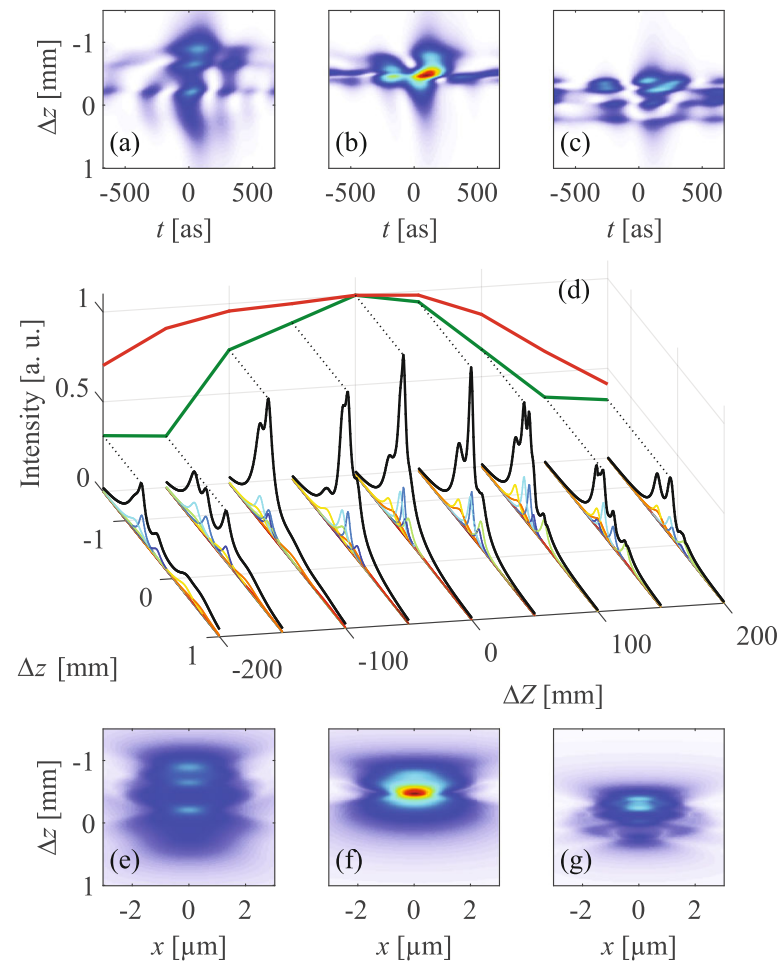

Figure 7: Estimated on-axis intensity as a function of time and $\Delta z$ for generation positions: $\Delta Z=-150 \mathrm{~mm}$ (a), $\Delta Z=0$ (b), and $\Delta Z=$ $150 \mathrm{~mm}$ (c). (d) Peak intensity (black) varying along the propagation axis $(\Delta z)$ for different generation positions $(\Delta Z)$. The harmonic intensities (multiplied by two) are plotted for each $\Delta Z$, going from order 11 (red) to 25 (blue). Measured total yield (red) and peak intensity (green) as a function of $\Delta Z$ are plotted in the intensity- $\Delta Z$-plane. Estimated peak intensity as a function of the transverse coordinate $x$ and $\Delta z$ for generation positions: $\Delta Z=-150 \mathrm{~mm}$ (e), $\Delta Z=0$ (f), and $\Delta Z=150 \mathrm{~mm}$ (g).

where $I_{q}$ are the relative experimental harmonic intensities. The phases, $\phi_{q}$, are the sum of the Gouy phase obtained by using the experimentally determined position of the focus and Rayleigh length for each harmonic and the inherent phase due to the generation process (see Equation (1)), also called attochirp [51].

In Figures 7(a)-7(c), we show the estimated attosecond pulse intensity on axis in the application chamber, as a function of time and position along the propagation axis $(\Delta z)$, for three generation positions, $\Delta Z=-150 \mathrm{~mm}$ (a), $\Delta Z=0$ (b), and $\Delta Z=150 \mathrm{~mm}$ (c). This is obtained using Equation (4) for each $\Delta z$ and taking the absolute square to get the intensity. The peak of the attosecond pulse intensity is extracted from these results and shown in Figure 7(d) in black as a function of $\Delta z$, for different generation positions $(\Delta Z)$. The intensity variation for each harmonic is marked in color going from the $11^{\text {th }}$ (red) to $25^{\text {th }}$ (blue). Figure $7(\mathrm{~d}$ ) also shows the maximum XUV peak intensity (green) and the total yield (red) for each generation position. Furthermore, the intensity distribution as a function of the transverse coordinate $x$ and $\Delta z$ is shown for $\Delta Z=-150 \mathrm{~mm}$ (e), $\Delta Z=0$ (f), and $\Delta Z=150 \mathrm{~mm}(\mathrm{~g})$.
As seen in Figure 7, the distribution of harmonic focus positions is clearly broader when the cell is before the IR focus (negative $\Delta Z$ ), as shown in panels (a) and (e). In contrast, the foci are closer to each other along the propagation axis when the cell is located at the IR focus or beyond, as shown in panels $(b, c)$ and (f, g). The maximum intensity is obtained for $\Delta Z=0$ and $\Delta z=-0.47 \mathrm{~mm}$, as seen in panels (b) and (f). When $\Delta Z<0$, the harmonics are focused at different positions, while when $\Delta Z>0$, the generation efficiency decreases. The total yield and the maximum intensity vary differently with respect to the generation position, as shown in Figure 7(d).

From the estimated spatiotemporal intensities in Figure 7 , we find for the conditions where the maximum intensity is obtained ( $\Delta Z=0$ and $\Delta z=-0.47 \mathrm{~mm}$ ), an average pulse duration of the individual attosecond pulses of $\sim 200$ as and a beam waist of $\sim 1.5 \mu \mathrm{m}$. Combining this with the XUV pulse energy, measured using an X-ray CCD camera (Andor) to be $1.6 \mathrm{~nJ}$, and a harmonic pulse duration of $20 \mathrm{fs}$ (half of the IR pulse duration, according to the same power law used for the harmonic beam widths above), gives an estimate of the maximum XUV peak intensity of $10^{13} \mathrm{~W} / \mathrm{cm}^{2}$. This corresponds to an intensity of 1 on the vertical axis in Figure 7(d).

\section{Conclusion}

By inserting a knife before and after the focus and scanning it through the XUV beam, we have mapped out the focus position and estimated the waist size of each individual harmonic, as a function of the position of the gas cell relative to the IR focus. The key conclusion of this work is that the harmonics' focusing properties are found to depend on the order and the generation position. We interpret this effect as a consequence of the intensity-dependent dipole phase. The experimental data agree with relatively simple simulations based on an analytical expression for the dipole phase $[34,35]$, the assumption that the harmonics can be represented as Gaussian beams [33], and without taking into account propagation effects in the nonlinear medium. These measurements allow us to estimate the focused intensity of the individual harmonics and the attosecond pulses in the train and to define the optimal conditions to achieve highintensity attosecond illumination. We find that these optimal conditions do not necessarily coincide with those leading to the highest yield. Even when the yield is approximately constant, the harmonic focusing properties can change significantly and thus affect the pulse duration and intensity of the attosecond pulses.

\section{Data Availability}

All data discussed in the article will be made available upon request.

\section{Disclosure}

Hugo Dacasa's present address is Department of Physical Chemistry, Complutense University of Madrid, Ciudad 
Universitaria, 28040 Madrid, Spain. Kévin Veyrinas' present address is Amplitude Laser Group, 11, avenue de Canteranne, Cité de la Photonique, 33600 Pessac, France. Hampus Wikmark's present address is Department of Physics and Astronomy, Uppsala University, Box 516, SE-751 20, Uppsala, Sweden. Sylvain Maclot's present address is Department of Physics, Gothenburg University, Box 100, 40530 Gothenburg, Sweden.

\section{Conflicts of Interest}

The authors declare no conflict of interest.

\section{Authors' Contributions}

M.H, J.P., M.P., and H.D. performed the experiments. P.E.-J, J.P., and C.A. designed the experiment. K.V., E.C., H.W., and S.M. contributed to building the experimental setup and preparing the experiment. M.H. did the data analysis and the simulations based on theory that P.S., H.W., C.G., C.A., and A.L. developed. M.H., A.L., P.E.-J., J.P., and M.P. wrote the article, with feedback from all the authors.

\section{Acknowledgments}

The authors acknowledge support from the Swedish Research Council, the European Research Council (advanced grant QPAP), the Knut and Alice Wallenberg Foundation, and the Crafoord Foundation. The research leading to these results has received funding from LASERLAB-EUROPE (grant agreement no. 654148, European Union's Horizon 2020 research and innovation programme). S.M. acknowledges financial support from the COST Action CA18212Molecular Dynamics in the GAS phase (MD-GAS), supported by COST (European Cooperation in Science and Technology).

\section{References}

[1] P. Agostini and L. F. DiMauro, "The physics of attosecond light pulses," Reports on Progress in Physics, vol. 67, no. 6, pp. 813-855, 2004.

[2] J. Tate, T. Auguste, H. G. Muller, P. Salières, P. Agostini, and L. F. DiMauro, "Scaling of wave-packet dynamics in an intense midinfrared field," Physical Review Letters, vol. 98, no. 1, article 013901, 2007.

[3] A. D. Shiner, C. Trallero-Herrero, N. Kajumba et al., "Wavelength scaling of high harmonic generation efficiency," Physical Review Letters, vol. 103, no. 7, article 073902, 2009.

[4] Y. Kobayashi, T. Ohno, T. Sekikawa, Y. Nabekawa, and S. Watanabe, "Pulse width measurement of high-order harmonics by autocorrelation," Applied Physics B: Lasers and Optics, vol. 70, no. 3, pp. 389-394, 2000.

[5] J.-F. Hergott, M. Kovacev, H. Merdji et al., "Extreme-ultraviolet high-order harmonic pulses in the microjoule range," Physical Review A, vol. 66, no. 2, article 021801, 2002.

[6] P. Rudawski, C. M. Heyl, F. Brizuela et al., "A high-flux highorder harmonic source," Review of Scientific Instruments, vol. 84, no. 7, article 073103, 2013.
[7] B. Manschwetus, L. Rading, F. Campi et al., "Two-photon double ionization of neon using an intense attosecond pulse train," Physical Review A, vol. 93, no. 6, article 061402, 2016.

[8] I. Makos, I. Orfanos, A. Nayak et al., "A 10-gigawatt attosecond source for non-linear XUV optics and XUV-pumpXUV- probe studies," Scientific Reports, vol. 10, no. 1, p. $3759,2020$.

[9] K. Midorikawa, Y. Nabekawa, and A. Suda, "XUV multiphoton processes with intense high-order harmonics," Progress in Quantum Electronics, vol. 32, no. 2, pp. 43-88, 2008.

[10] E. J. Takahashi, P. Lan, O. D. Mücke, Y. Nabekawa, and K. Midorikawa, "Attosecond nonlinear optics using gigawattscale isolated attosecond pulses," Nature Communications, vol. 4 , no. 1, 2013.

[11] A. Nayak, I. Orfanos, I. Makos et al., "Multiple ionization of argon via multi-xuv-photon absorption induced by 20 -gw high-order harmonic laser pulses," Physical Review A, vol. 98, no. 2, article 023426, 2018.

[12] B. Senfftleben, M. Kretschmar, A. Hoffmann et al., "Highly nonlinear ionization of atoms induced by intense high-harmonic pulses," Journal of Physics: Photonics, vol. 2, no. 3, 2020.

[13] P. A. Carpeggiani, P. Tzallas, A. Palacios, D. Gray, F. Martín, and D. Charalambidis, "Disclosing intrinsic molecular dynamics on the 1-fs scale through extreme-ultraviolet pump-probe measurements," Physical Review A, vol. 89, no. 2, article 023420, 2014.

[14] P. Tzallas, D. Charalambidis, N. A. Papadogiannis, K. Witte, and G. D. Tsakiris, "Direct observation of attosecond light bunching," Nature, vol. 426, no. 6964, pp. 267-271, 2003.

[15] Y. Nabekawa, H. Hasegawa, E. J. Takahashi, and K. Midorikawa, "Production of doubly charged helium ions by two-photon absorption of an intense sub-10-fs soft X-ray pulse at $42 \mathrm{eV}$ photon energy," Physical Review Letters, vol. 94, no. 4, article 043001, 2005.

[16] C. Pellegrini, A. Marinelli, and S. Reiche, "The physics of X-ray free-electron lasers," Reviews of Modern Physics, vol. 88, no. 1, article 015006, 2016.

[17] L. Young, K. Ueda, M. Gühr et al., "Roadmap of ultrafast x-ray atomic and molecular physics," Journal of Physics B: Atomic, Molecular and Optical Physics, vol. 51, no. 3, p. 032003, 2018.

[18] J. Duris, S. Li, T. Driver et al., “Tunable isolated attosecond xray pulses with gigawatt peak power from a free-electron laser," Nature Photonics, vol. 14, no. 1, pp. 30-36, 2020.

[19] A. Suda, H. Mashiko, and K. Midorikawa, "Focusing intense high-order harmonics to a micron spot size," in Progress in Ultrafast Intense Laser Science II, pp. 183-198, Springer Berlin Heidelberg, Berlin, Heidelberg, 2007.

[20] H. Motoyama, T. Sato, A. Iwasaki et al., "Development of highorder harmonic focusing system based on ellipsoidal mirror," Review of Scientific Instruments, vol. 87, no. 5, article 051803, 2016.

[21] C. Valentin, D. Douillet, S. Kazamias et al., "Imaging and quality assessment of high-harmonic focal spots," Optics Letters, vol. 28, no. 12, pp. 1049-1051, 2003.

[22] F. Frassetto, S. Anumula, F. Calegari, A. Trabattoni, M. Nisoli, and L. Poletto, "Microfocusing beamline for xuv-xuv pumpprobe experiments using hh generation," in Adaptive X-Ray Optics III, vol. 9208, pp. 159-167, September 2014.

[23] H. Coudert-Alteirac, H. Dacasa, F. Campi et al., "Micro-focusing of broadband highorder harmonic radiation by a double toroidal mirror," Applied Sciences, vol. 7, no. 11, p. 1159, 2017. 
[24] I. Orfanos, I. Makos, I. Liontos et al., "Non-linear processes in the extreme ultraviolet," Journal of Physics: Photonics, vol. 2, no. $4,2020$.

[25] J. A. Arnaud, W. M. Hubbard, G. D. Mandeville, B. de la Clavière, E. A. Franke, and J. M. Franke, "Technique for fast measurement of Gaussian laser beam parameters," Applied Optics, vol. 10, no. 12, pp. 2775-2776, 1971.

[26] D. R. Skinner and R. E. Whitcher, "Measurement of the radius of a high-power laser beam near the focus of a lens," Journal of Physics E: Scientific Instruments, vol. 5, no. 3, pp. 237-238, 1972.

[27] L. le Déroff, P. Salières, and B. Carré, "Beam-quality measurement of a focused high-order harmonic beam," Optics Letters, vol. 23, no. 19, pp. 1544-1546, 1998.

[28] D. G. Lee, J. J. Park, J. H. Sung, and C. H. Nam, "Wave-front phase measurements of high-order harmonic beams by use of point-diffraction interferometry," Optics Letters, vol. 28, no. 6, pp. 480-482, 2003.

[29] E. Frumker, G. G. Paulus, H. Niikura, D. M. Villeneuve, and P. B. Corkum, "Frequency-resolved high-harmonic wavefront characterization," Optics Letters, vol. 34, no. 19, pp. 30263028, 2009.

[30] D. R. Austin, T. Witting, C. A. Arrell et al., "Lateral shearing interferometry of high-harmonic wavefronts," Optics Letters, vol. 36, no. 10, pp. 1746-1748, 2011.

[31] H. Motoyama, A. Iwasaki, Y. Takei et al., "Broadband nanofocusing of high-order harmonics in soft $\mathrm{x}$-ray region with ellipsoidal mirror," Applied Physics Letters, vol. 114, no. 24, article 241102, 2019.

[32] D. T. Lloyd, K. O’Keeffe, and S. M. Hooker, "Complete spatial characterization of an optical wavefront using a variableseparation pinhole pair," Optics Letters, vol. 38, no. 7, pp. 1173-1175, 2013.

[33] E. Frumker, G. G. Paulus, H. Niikura, A. Naumov, D. M. Villeneuve, and P. B. Corkum, "Order-dependent structure of high harmonic wavefronts," Optics Express, vol. 20, no. 13, article 13870, p. 13877, 2012.

[34] H. Wikmark, C. Guo, J. Vogelsang et al., "Spatiotemporal coupling of attosecond pulses," Proceedings of the National Academy of Sciences, vol. 116, no. 11, pp. 4779-4787, 2019.

[35] L. Quintard, V. Strelkov, J. Vabek et al., “Optics-less focusing of XUV high-order harmonics," Science Advances, vol. 5, no. 4, article eaau7175, 2019.

[36] H. Dacasa, H. Coudert-Alteirac, C. Guo et al., "Single-shot extreme-ultraviolet wavefront measurements of high-order harmonics," Optics Express, vol. 27, no. 3, pp. 2656-2670, 2019.

[37] C. Hernández-García, A. Picón, J. San Román, and L. Plaja, "Attosecond extreme ultraviolet vortices from high-order harmonic generation," Physical Review Letters, vol. 111, no. 8, article 083602, 2013

[38] S. Chatziathanasiou, S. Kahaly, D. Charalambidis, P. Tzallas, and E. Skantzakis, "Imaging the source of high-harmonics generated in atomic gas media," Optics Express, vol. 27, no. 7, pp. 9733-9739, 2019.

[39] H. Wolter, "Spiegelsysteme streifenden Einfalls als abbildende Optiken für Röntgenstrahlen," Annalen der Physik, vol. 445, no. 1-2, pp. 94-114, 1952.

[40] P. B. Corkum, "Plasma perspective on strong field multiphoton ionization," Physical Review Letters, vol. 71, no. 13, pp. 1994-1997, 1993.
[41] K. J. Schafer, B. Yang, L. F. DiMauro, and K. C. Kulander, "Above threshold ionization beyond the high harmonic cutoff,” Physical Review Letters, vol. 70, no. 11, pp. 1599-1602, 1993.

[42] M. Bellini, C. Lyngå, A. Tozzi et al., "Temporal coherence of ultrashort high-order harmonic pulses," Physical Review Letters, vol. 81, no. 2, pp. 297-300, 1998.

[43] P. Salières, B. Carré, L. le Déroff et al., "Feynman's pathintegral approach for intense-laser-atom interactions," Science, vol. 292, no. 5518, pp. 902-905, 2001.

[44] M. Lewenstein, K. C. Kulander, K. J. Schafer, and P. H. Bucksbaum, "Rings in above-threshold ionization: a quasiclassical analysis," Physical Review A, vol. 51, no. 2, pp. 1495-1507, 1995.

[45] C. Guo, A. Harth, S. Carlström et al., "Phase control of attosecond pulses in a train," Journal of Physics B: Atomic Molecular and Optical Physics, vol. 51, no. 3, article 034006, 2018.

[46] C. Hernández-García, J. S. Román, L. Plaja, and A. Picón, "Quantum-path signatures in attosecond helical beams driven by optical vortices," New Journal of Physics, vol. 17, no. 9, article 093029, 2015.

[47] F. Catoire, A. Ferré, O. Hort et al., "Complex structure of spatially resolved high-order-harmonic spectra," Physical Review A, vol. 94, no. 6, 2016.

[48] L. Rego, J. S. Román, A. Picón, L. Plaja, and C. HernándezGarcía, "Nonperturbative twist in the generation of extremeultraviolet vortex beams," Physical Review Letters, vol. 117, no. $16,2016$.

[49] J. A. Pérez-Hernández, L. Roso, and L. Plaja, "Harmonic generation beyond the Strong-Field approximation: the physics behind the short-wave-infrared scaling laws," Optics Express, vol. 17, no. 12, pp. 9891-9903, 2009.

[50] C. G. Durfee, A. R. Rundquist, S. Backus, C. Herne, M. M. Murnane, and H. C. Kapteyn, "Phase matching of high-order harmonics in hollow waveguides," Physical Review Letters, vol. 83, no. 11, pp. 2187-2190, 1999.

[51] Y. Mairesse, A. de Bohan, L. J. Frasinski et al., "Attosecond synchronization of high-harmonic soft X-rays," Science, vol. 302 , no. 5650, pp. 1540-1543, 2003. 